\title{
Wave Propagation in Stochastic Spacetimes: Localization, Amplification and Particle Creation
}

\author{
B. L. Hu and K. Shiokawa \\ Department of Physics, University of Maryland, College Park, MD 20742, USA \\ (umdpp 97-125, submitted to Phys. Rev. D on July 29, 1997) \\ We study novel effects associated with electromagnetic wave propagation \\ in a Robertson-Walker universe and the Schwarzschild spacetime with a small \\ amount of metric stochasticity. We find that localization of electromagnetic \\ waves occurs in a Robertson-Walker universe with time-independent metric \\ stochasticity, while time-dependent metric stochasticity induces exponential \\ instability in the particle production rate. For the Schwarzschild metric, time- \\ independent randomness can decrease the total luminosity of Hawking radia- \\ tion due to multiple scattering of waves outside the black hole and gives rise to \\ event horizon fluctuations and thus fluctuations in the Hawking temperature.
}




\section{INTRODUCTION}

Wave propagation and localization [1] in a random media has been studied extensively for the last two decades [2 14. It is known that on the mesoscopic scale, classical wave propagation in a random media can be treated in a similar way as electron transport in a random potential [四]. One expects to see the diffusive, localization properties of classical waves similar to electrons moving in the presence of impurities [5,6]. Wave propagation in curved spacetime is an important topic both in general relativity [7] and in semiclassical gravity theory [8]. Classical scalar, electromagnetic and gravitational waves in FriedmannRobertson-Walker (FRW) Universe probe into the state of the universe and manifest in basic cosmological processes such as structure and defect formation, while that in the Schwarzschild and Kerr spacetimes depict high energy astrophysical processes in black holes. The secondquantized version in terms of quantum fields gives rise to cosmological particle creation [9] and Hawking radiation [10] which are important processes in the early universe and black holes collapse. Recent progress in studying Planck energy processes, especially the backreaction effect of quantum fields in curved spacetime, underscores the importance of including fluctuations in particle creation [11, [12] and the associated energy momentum tensor

of quantum fields [13 15], and fluctuations and dissipation in the dynamics of spacetime [16] 19.

The program devoted to quantum matter field and classical background spacetime with metric fluctuations mentioned above is rather involved, because it requires the calculation of four point functions [20] and demands a self-consistent solution [21,22]. In this paper, as a useful parallel, we attempt to address an easier problem, that of wave propagation in a stochastic spacetime. It is designed to highlight the effect of fluctuations in a background metric, while not demanding an explanation of their source, or their mutual influence. Stochastic components in the metric can be induced by primordial gravitational waves, 
topological defects in the sub-Planckian scale, or intrisic metric fluctuations of background spacetimes at the Planck scale. Their detection and analysis can provide valuable information about the state of the early universe and black holes. As distinct from the self-consistent treatment which is necessary for Planck scale processes, wave propagation in curved spacetimes with metric stochasticity is a test-field treatment. It is nonetheless still a useful probe for fluctuations in sub-Planckian processes (such as GUT scale phase transition), which could have left important imprints on the observable universe [23].

In this paper, we wish to study novel effects associated with electromagnetic waves propagation in the Friedmann-Robertson-Walker (FRW) universes and the Schwarzschild spacetime with a small amount of metric stochasticity. Here we employ a useful observation to link up with the more familiar subject of wave propagation in random media studied extensively in condensed matter and mesoscopic physics [5,6]. We first show the formal equivalence of the wave equations in curved spacetimes with wave propagation in a media in flat space and identify how the metric components appear in the permittivity function (or refractive index) of the media [24]. Then we introduce metric fluctuations as a stochastic component in the permittivity function and study wave propagation in a curved spacetime with metric stochasticity as if it were in a random media. In a spherically symmetric spacetime the wave equation for the radial part can be written in the form of a Schrödinger equation in one dimension. The effect of the curvature of spacetime appears in the potential term in the equation. Once the wave equation is reduced to the parametric form with a stochastic component, the familiar methods used in quantum field theory in curved spacetime and insights accumulated in mesoscopic physics can work in each other's advantage. We analyse in detail the cases with time-independent and time-dependent metric stochasticity and find that localization of electromagnetic waves occurs in a metric with time-independent fluctuations. In cases where there are time-dependent randomness in the metric, exponential instability in the particle production rate occurs. These are new effects due exclusively to the presence 
of metric fluctuations in the background spacetimes. For the Schwarzschild metric, timeindependent randomness outside the horizon will decrease the total luminosity of Hawking radiation due to multiple scattering of waves. if the randomness reaches the horizon, it contributes to the fluctuations of Hawking temperature. Time-dependent stochasticity is a more complicated matter which requires a self-consistent analysis of the interaction between spacetime and the waves or fields as encoded in the fluctuation-dissipation relations [21,22]. This is to be investigated later as a part of the stochastic backreaction problem mentioned above.

The paper is organized as follows: In Sec. 2, we show how an electromagnetic wave propagating in curved spacetime can be related to that in flat space but with a refractive index depending on the metric components [24]. This section is meant to be a short cut for readers not too familar with curved spacetime physics to see the correspondence with wave phenonema. (Readers familiar with it can skip to Sec. 3). In Section 3, using the methods developed in Sec. 2 we study wave propagation in curved spacetime where the metric has a stochastic component. We use the Friedmann-Robertson-Walker universe as an example to show that if the stochastic component of the metric is independent of time, and for a sufficiently smooth randomness the Maxwell equation has the same form as a conformally coupled scalar wave equation. If the randomness appears only in the radial direction, the radial wave equation has the same form as an one dimensional Schrödinger equation in a random potential. Because the electromagnetic wave equation is conformally invariant, it bears the same form as in flat space. With a stochastic metric component we see that wave localizes in space. In Sec. 4, we study the case of time-dependent (but space-independent) stochasticity in the metric, and show that parametric amplification takes place giving rise to cosmological particle creation. However, because of the metric fluctuations the rate of this amplification increases exponentially in time. The fluctuations in the particle creation rate is also discussed. In Sec. 5, we study wave propagation in a Schwarzschild spacetime 
with time-independent fluctuations. We show that this can decrease the total luminosity of Hawking radiation due to multiple scattering of waves outside the black hole. Fluctuations which involve event horizon will give rise to fluctuations in the Hawking temperature. In Sec. 6 we summarize our findings and end with a short discussion.

\section{CLASSICAL ELECTROMAGNETIC WAVES IN CURVED SPACETIME}

Maxwell's equations for an electromagnetic field tensor $F_{\mu \nu}$ in a gravitational field with metric $g_{\mu \nu}$ are given by

$$
F_{\nu}^{\mu \nu}=0 \quad F_{\mu \nu ; \sigma}+F_{\nu \sigma ; \mu}+F_{\sigma \mu ; \nu}=0
$$

where semicolons denote covariant derivatives with respect to $g_{\mu \nu}$. These equations can be cast in a form for waves propagating in a permeable media in flat space [24]. The

correspondence between $E, H$ (the electric field and magnetic induction) and $D, B$ (the electric displacement and magnetic field) respectively is given by

$$
\begin{gathered}
D_{i}=\epsilon_{i k} E_{k}-(G \times H)_{i} \\
B_{i}=\mu_{i k} H_{k}-(G \times E)_{i}
\end{gathered}
$$

where the dielectric and permitivity functions are

$$
\begin{aligned}
\epsilon_{i k} & =\mu_{i k}=-(-g)^{1 / 2} \frac{g^{i k}}{g_{00}} \\
G_{i} & =-\frac{g^{0 i}}{g_{00}} .
\end{aligned}
$$

\section{A. Friedmann-Robertson-Walker Universe}

Let us now consider the Friedmann-Robertson-Walker (FRW) spacetimes with line elements 


$$
-d s^{2}=-d t^{2}+a(t)^{2} R^{2}\left[d \chi^{2}+s^{2}(\chi)\left(d \theta^{2}+\sin ^{2} \theta d \phi^{2}\right)\right]
$$

where $s(\chi)=\sin \chi, \chi, \sinh \chi$ correspond to closed, flat, and open cases respectively. Here $a(t)$ is the scale factor and $R$ is the radius at time $t_{0}$ where $a\left(t_{0}\right)=1$.

Using Cartesian coodinates,

$$
\begin{aligned}
& d t=a(t) d \eta \\
& x^{1}=2 R t(\chi / 2) \sin \theta \cos \phi \\
& x^{2}=2 R t(\chi / 2) \sin \theta \sin \phi \\
& x^{3}=2 R t(\chi / 2) \cos \theta
\end{aligned}
$$

where $\eta \equiv \int d t / a$ is the conformal time, and $t(\chi / 2)=\tan (\chi / 2), \chi / 2, \tanh (\chi / 2)$ correspond to the closed, flat, and open cases respectively. We can then write the FRW line element in the form

$$
-d s^{2}=a^{2}\left[-d \eta^{2}+f^{2}(\rho)\left(\delta_{i j} d x^{i} d x^{j}\right)\right]
$$

where

$$
\begin{aligned}
\rho & =\left[\sum_{i}\left(x^{i}\right)^{2}\right]^{1 / 2} \\
& =2 R t(\chi / 2)
\end{aligned}
$$

and

$$
f(\rho)=\left(\begin{array}{c}
\frac{1}{1+\rho^{2} / 4 R^{2}} \\
1 \\
\frac{1}{1-\rho^{2} / 4 R^{2}}
\end{array}\right)
$$

where the column elements correspond to closed, flat, and open cases respectively.

In this isotropic form of the metric, the Maxwell equations are given by 


$$
\begin{aligned}
\nabla \times \vec{E} & =-\frac{d}{d \eta}(f(\rho) \vec{H}) \\
\nabla \times \vec{H} & =\frac{d}{d \eta}(f(\rho) \vec{E}) \\
\nabla \cdot(f \vec{E}) & =0 \\
\nabla \cdot(f \vec{H}) & =0
\end{aligned}
$$

where one can identify the dielectric and permittivity functions as

$$
\begin{aligned}
\epsilon_{i k} & =\mu_{i k}=f(\rho) \delta_{i k} \\
G_{i} & =0 .
\end{aligned}
$$

Assuming a harmonic (conformal) time dependence of the solution $e^{-i \omega \eta}$, we can write (II.8) as

$$
\begin{aligned}
\nabla \times \vec{E} & =i \omega f(\rho) \vec{H} \\
\nabla \times \vec{H} & =-i \omega f(\rho) \vec{E} \\
\nabla \cdot(f \vec{E}) & =0 \\
\nabla \cdot(f \vec{H}) & =0 .
\end{aligned}
$$

The symmetry in the relation ([I.10) allows us to write this in the more compact form

$$
\begin{aligned}
\nabla \times \vec{F} & =\omega f(\rho) \vec{F} \\
\nabla \cdot(f \vec{F}) & =0
\end{aligned}
$$

where $\vec{F} \equiv \vec{E}+i \vec{H}$.

For the FRW metric with spherical symmetry the solutions can be expressed in terms of the vector spherical harmonics $\vec{Y}_{l m}(\theta, \phi)$ obtained by operating on the scalar spherical harmonics $Y_{l m}(\theta, \phi)$ with the invariant operator $-i r \cdot \nabla$ as

$$
\begin{aligned}
\vec{E} & =\sum_{l, m}\left[\frac{i}{\omega f} A_{l m}^{E} \nabla \times g_{l}^{E}(\rho) \vec{Y}_{l m}+A_{l m}^{M} g_{l}^{M}(\rho) \vec{Y}_{l m}\right] \\
\vec{H} & =\sum_{l, m}\left[A_{l m}^{E} g_{l}^{E}(\rho) \vec{Y}_{l m}-\frac{i}{\omega f} A_{l m}^{M} \nabla \times g_{l}^{M}(\rho) \vec{Y}_{l m}\right]
\end{aligned}
$$


where $g_{l}^{E, M}(\rho)$ are functions of radial distance only and $A_{l m}^{E, M}$ are the coefficients. Superscripts $E$ and $M$ denote the electric and magnetic multipole field components, respectively.

The field $\vec{F}$ is given by

$$
\begin{aligned}
\vec{F} & =\sum_{l, m}\left[i A_{l m}^{E}\left(\frac{1}{\omega f} \nabla \times g_{l}^{E}(\rho) \vec{Y}_{l m}+g_{l}^{E}(\rho) \vec{Y}_{l m}\right)\right. \\
& \left.+A_{l m}^{M}\left(g_{l}^{M}(\rho) \vec{Y}_{l m}+\frac{1}{\omega f} \nabla \times g_{l}^{M}(\rho) \vec{Y}_{l m}\right)\right] .
\end{aligned}
$$

One can identify the $(l, m)$ component of a magnetic multipole field $\vec{F}_{l m}^{M}$ as

$$
\vec{F}_{l m}^{M}=g_{l}^{M}(\rho) \vec{Y}_{l m}+\frac{1}{\omega f} \nabla \times g_{l}^{M}(\rho) \vec{Y}_{l m} .
$$

Writing the magnetic multipole scalar field function

$$
\psi^{M}(\chi)=R \rho(\chi) g_{l}^{M}(\rho(\chi))
$$

we obtain the radial equation for $\psi^{M}$ as

$$
\frac{d^{2} \psi^{M}(\chi)}{d \chi^{2}}+\left(n^{2}-U(\chi)\right) \psi^{M}(\chi)=0
$$

where $n=R \omega$ and

$$
U(\chi)=\frac{j(j+1)}{s^{2}(\chi)}
$$

\section{B. Schwarzschild Spacetime}

The line element for the Schwarzschild spacetime is given by

$$
-d s^{2}=-\left(1-\frac{2 M}{r}\right) d t^{2}+\left(1-\frac{2 M}{r}\right)^{-1} d r^{2}+r^{2}\left[d \theta^{2}+\sin ^{2} \theta d \phi^{2}\right] .
$$

If we define $r \equiv \rho+M+M^{2} / 4 \rho$, we can write (IIII.35) as

$$
-d s^{2}=-f_{1}(\rho) d t^{2}+f_{2}^{2}(\rho)\left[d \rho^{2}+\rho^{2} d \theta^{2}+\rho^{2} \sin ^{2} \theta d \phi^{2}\right]
$$


where $f_{1}(\rho)=1-\frac{2 M}{r}$ and $f_{2}(\rho)=r / \rho$. Furthermore, a spatial coordinate transformation

$$
\begin{aligned}
& x^{1}=\rho \sin \theta \cos \phi \\
& x^{2}=\rho \sin \theta \sin \phi \\
& x^{3}=\rho \cos \theta
\end{aligned}
$$

brings (II.19) into a Cartesian form

$$
-d s^{2}=-f_{1}(\rho) d t^{2}+f_{2}^{2}(\rho)\left[\left(d x^{1}\right)^{2}+\left(d x^{2}\right)^{2}+\left(d x^{3}\right)^{2}\right] .
$$

Considering this spacetime acting as a medium, the corresponding dielectric and permittivity functions are expressed in the same form as in ([1.9) with

$$
f(\rho)=\frac{f_{2}(\rho)}{\left(f_{1}(\rho)\right)^{1 / 2}} .
$$

In such a medium, the radial equation for the magnetic multipole field is 25]

$$
\frac{d^{2} \psi^{M}(r *)}{d r *^{2}}+\left[\omega^{2}-U(r *)\right] \psi^{M}(r *)=0
$$

where $r *=r+2 M \log (r / 2 M-1)$ is the Regge-Wheeler (turtoise) coordinate, and the potential term is given by

$$
U(r *)=\frac{j(j+1)}{r^{2}(r *)}\left[1-\frac{2 M}{r(r *)}\right] .
$$

\section{WAVE PROPAGATION IN CURVED SPACETIME WITH METRIC STOCHASTICITY}

Consider the metric as containing an averaged (deterministic) part $g_{\mu \nu}^{(0)}$ and a small fluctuating (stochastic) part $h_{\mu \nu}$

$$
g_{\mu \nu}=g_{\mu \nu}^{(0)}+h_{\mu \nu}
$$


then from (II.3)

$$
\begin{aligned}
\epsilon_{i k} & =\mu_{i k}=-\frac{\left(-g^{(0)}\right)^{1 / 2}}{g_{00}^{(0)}} g^{(0) i k}+\frac{\left(-g^{(0)}\right)^{1 / 2}}{g_{00}^{(0)}}\left(h^{i j}-\frac{1}{2} h g^{(0) i k}\right) \\
& =\epsilon_{i k}^{(0)}+\delta \epsilon_{i k}
\end{aligned}
$$

where we have used the synchronous $h_{00}=0$ gauge [26]. The traceless $h \equiv \sum_{i=1}^{3} h_{i}^{i}=0$ gauge further simplifies the expression and we obtain the correlation function of the fluctuating part of the refractive index in terms of the metric fluctuations as

$$
\left\langle\delta \epsilon_{i j}(x) \delta \epsilon_{k l}\left(x^{\prime}\right)\right\rangle=\frac{\left(-g^{(0)}(x)\right)^{1 / 2}\left(-g^{(0)}\left(x^{\prime}\right)\right)^{1 / 2}}{g_{00}^{(0)}(x) g_{00}^{(0)}\left(x^{\prime}\right)}\left\langle h^{i j}(x) h^{k l}\left(x^{\prime}\right)\right\rangle .
$$

For flat spacetime, it has the simple form

$$
\left\langle\delta \epsilon_{i j}(x) \delta \epsilon_{k l}\left(x^{\prime}\right)\right\rangle=\left\langle h^{i j}(x) h^{k l}\left(x^{\prime}\right)\right\rangle
$$

We see metric fluctuations can thus be represented as fluctuations in the optical index.

\section{A. Time Independent Metric Stochasticity}

In the rest of this section, we consider cases in which the permitivity function contains only spatial disorder.

If we assume the background metric as well as the stochasticity are both isotropic, we can write the corresponding refractive index as

$$
\begin{aligned}
\epsilon_{i k} & =\mu_{i k}=f(\vec{x}) \delta_{i k} \\
G_{i} & =0
\end{aligned}
$$

where $f(\vec{x})$ is a random variable.

Assuming a harmonic time dependence of the field with frequency $\omega$, Eq. (II.11) with source term $\vec{J}$ becomes 


$$
(\vec{L}-\omega f(\vec{x})) \vec{F}=\vec{J}
$$

where $\vec{L} \equiv \nabla \times$. Define Green's function $g\left(x, x^{\prime}\right)$ as

$$
(\vec{L}-\omega f(\vec{x})) g\left(x, x^{\prime}\right)=\delta\left(x-x^{\prime}\right) .
$$

Then the disorder-averaged Green's function is

$$
\begin{aligned}
G\left(x, x^{\prime}\right) & \equiv\left\langle g\left(x, x^{\prime}\right)\right\rangle \\
& =\left\langle(\vec{L}-\omega f(\vec{x}))^{-1}\right\rangle .
\end{aligned}
$$

If we define the mean field Green's function $G^{(0)}\left(x, x^{\prime}\right)$ as

$$
G^{(0)}\left(x, x^{\prime}\right) \equiv(\vec{L}-\omega\langle f(\vec{x})\rangle)^{-1}
$$

we obtain the Lippman-Schwinger equation

$$
G^{-1}\left(x, x^{\prime}\right)=G^{(0)-1}\left(x, x^{\prime}\right)+\omega \Sigma\left(x, x^{\prime}\right)
$$

where $\Sigma\left(x, x^{\prime}\right)$ is the self-energy. Also averaging ([II.6), we have

$$
\langle\vec{L} \vec{F}\rangle-\omega\langle f(\vec{x}) \vec{F}\rangle=\vec{J}
$$

Define $\overrightarrow{\mathcal{F}} \equiv f \vec{F}=\vec{D}+i \vec{B}$, then from (III.11)

$$
\begin{aligned}
\omega\langle\overrightarrow{\mathcal{F}}\rangle & =\langle\vec{L} \vec{F}\rangle-\vec{J} \\
& =\int d x^{\prime}\left(G^{(0)-1}\left(x, x^{\prime}\right)+\omega \Sigma\left(x, x^{\prime}\right)-\vec{L}\right)\langle\vec{F}\rangle \\
\langle\overrightarrow{\mathcal{F}}(x)\rangle & =\int d x^{\prime}\left(\langle f(\vec{x})\rangle \delta\left(x-x^{\prime}\right)-\Sigma\left(x, x^{\prime}\right)\right)\left\langle\vec{F}\left(x^{\prime}\right)\right\rangle .
\end{aligned}
$$

We see that the complex electromagnetic displacement vector has a nonlocal dependence on the media due to the self-energy term. We can thus develop a formalism making use 
of techniques from quantum field theory to study the effect of dissipation, higher order correlation, screening, etc. (see e.g., [27]). This is a subject for future investigation.

Further simplification arises in cases when the polarization effects are negligible. From ([1.10), we obtain

$$
\nabla^{2} \vec{E}+\omega^{2} f^{2}(\vec{x}) \vec{E}+\nabla \log f(\vec{x}) \times \nabla \times \vec{E}+\nabla((\vec{E} \cdot \nabla) \log f(\vec{x}))=0
$$

Under the conditions that the inhomogeneity and disorder in $f$ are smooth, namely, $\omega R>>1$ and $\omega \lambda>>1$, where $\lambda$ is the characteristic length scale of the disorder, the third and the fourth term representing polarization effects are negligible. (III.14) then reduces to

$$
\nabla^{2} \vec{E}+\omega^{2} f^{2}(\vec{x}) \vec{E}=0
$$

Then each component satisfies the scalar wave equation

$$
\nabla^{2} \phi+\omega^{2} f^{2}(\vec{x}) \phi=0
$$

Now consider the conformal type of stochasticity only in the spatial part of the metric of the form 円

$$
f(\vec{x})=f(\rho) e^{g \sigma(\vec{x})}
$$

where $\sigma(\vec{x})$ is a stochastic function and $g$ is a small parameter. Expanding it with respect to $g$ as

$$
f(\vec{x})=f(\rho)(1+g \sigma(\vec{x}))
$$

(III.16) becomes

\footnotetext{
1 The conformal invariance of Maxwell's equations implies that the conformal fluctuations in the spacetime metric can be factorized away, and hence they do not give rise to particle creation [28].
} 


$$
\nabla^{2} \phi+\omega^{2} f^{2}(\rho) \phi+2 g \omega^{2} f^{2}(\rho) \sigma(\vec{x}) \phi=0
$$

In a flat FRW spacetime $f(\rho)=1$, or, for $\rho<<R$ in closed and open cases, $f(\rho) \sim 1$. In this region, all three cases are described by the equation in flat spacetime

$$
\nabla^{2} \phi+\omega^{2} \phi+2 g \omega^{2} \sigma \phi=0
$$

The corresponding equation for the Green function is

$$
\left(\nabla^{2}+\omega^{2}+2 g \omega^{2} \sigma\right) G\left(\vec{x}, \overrightarrow{x^{\prime}}, \omega\right)=\delta\left(\vec{x}-\overrightarrow{x^{\prime}}\right) .
$$

The disorder-averaged Green function $\left\langle G\left(\vec{k}, \overrightarrow{k^{\prime}}, \omega\right)\right\rangle=G(\vec{k}, \omega) \delta_{k k^{\prime}}$ is known to have a form

$$
G(\vec{k}, \omega)=\frac{1}{\omega^{2}-\vec{k}^{2}+i \frac{\omega}{l}}
$$

in the leading order in $(\omega l)^{-1}$, where $l$ is an elastic mean free path. For a Gaussian white noise (local) random variable $\sigma(\vec{x}), l=2 \pi / g D \omega^{4}$ where $D$ is the strength of disorder defined by $\left\langle\sigma(\vec{x}) \sigma\left(\overrightarrow{x^{\prime}}\right)\right\rangle=D \delta(\vec{x}-\vec{x})$.

We also define the intensity

$$
I(\vec{x})=\left\langle\phi^{*}(\vec{x}) \phi\left(\overrightarrow{x^{\prime}}\right)\right\rangle
$$

and the intensity-intensity correlation function

$$
C\left(\vec{x}, \overrightarrow{x^{\prime}}\right)=\left\langle\phi^{*}(\vec{x}) \phi(\vec{x}) \phi^{*}\left(\overrightarrow{x^{\prime}}\right) \phi\left(\overrightarrow{x^{\prime}}\right)\right\rangle_{C}
$$

where $C$ denotes the cumulant. As shown in [29], we can evaluate the disorder-averaged intensity as

$$
\langle I(\vec{x})\rangle=\frac{3}{16 \pi^{2} l|\vec{x}|} .
$$

If we confine the disorder in a slab of thickness $L$, and cross-sectional area $A$, then for $r \equiv\left|\vec{x}-\overrightarrow{x^{\prime}}\right|<l$, 


$$
C\left(\vec{x}, \overrightarrow{x^{\prime}}\right)=\langle I(\vec{x})\rangle\left\langle I\left(\overrightarrow{x^{\prime}}\right)\right\rangle\left(\frac{\sin \omega r}{\omega r}\right)^{2} e^{-\frac{r}{l}}
$$

For $r>l$, diffusion modes interact with each other and give rise to a long range correlation

with a power law decay 30]. In the Schrödinger equation analogy, this corresponds to conductance fluctuations in metals [31.

\section{B. Radial Disorder - Localization}

Here we also start from the conformal type of metric stochasticity but assume that the stochasticity is only in the radial direction

$$
f(x)=\frac{1}{1+t^{2}(\chi / 2)} e^{g \sigma(\chi)}
$$

where $g$ is a small parameter ( Such kind of randomness may arise from averaging out more inhomogeneous types of randomness in a small spatial region due to the spherically symmetric nature of the underlying curved spacetime).

If $\sigma(\chi)$ varies slowly in space $\left(\sigma^{\prime}<<\sigma\right)$, the wave equation in the radial direction (II.16) is replaced by

$$
\frac{d^{2} \psi}{d \chi^{2}}+\left[n^{2}(1+g \sigma)^{2}-\frac{j(j+1)}{s^{2}(\chi)}\right] \psi=0
$$

where we simply use $\psi$ for $\psi^{M}$. This has the same form as a scalar wave equation in a media with a random refractive index.

For the closed FRW universe, the angular-momentum induced potential term in (III.28) is a single well potential which becomes infinitely high at the origin and $\chi=\pi$. Then the wave is necessarily localized around the bottom of the well in this coordinate. On the other hand, for the flat and open FRW universe cases, the potential term becomes asymptotically flat as the radius becomes infinite. In these cases, the stochastic term plays a significant role in the transport property of the wave. If we expand the second term in (III.28) into 
$n^{2}+2 g n^{2} \sigma$ around the perturbation parameter $g$ and note the positivity of the original second term, we see ([11.28) describes a Schrödinger equation with a fixed positive energy. As such, we can write (III.28) as

$$
-\frac{d^{2} \psi}{d \chi^{2}}+V(\chi) \psi=E \psi
$$

where $E \equiv n^{2}$ and $V(\chi) \equiv-2 g n^{2} \sigma+\left[j(j+1) / s^{2}(\chi)\right]$.

For the flat or open universes, the potential barrier vanishes in the asymptotic limit. The property of the eigenfunction in this case is well-studied from the context of electron transport 田 6 . It is known that in one dimension for any value of $E$, the eigenstate of Eq. ([11.29) localizes [1, [. N. Namely, all eigenfunctions decay exponentially with rate given by the Lyapunov exponent [see ([II.32) below]. If the correlation radius $\chi_{c}$ of the fluctuating part of the potential $V(\chi)$ is smaller than the "wavelength" $2\left(\chi_{c}<<n^{-1}\right)$, the potential can be considered as the white noise type

$$
\left\langle V(\chi) V\left(\chi^{\prime}\right)\right\rangle=4 g^{2} n^{4}\left\langle\sigma(\chi) \sigma\left(\chi^{\prime}\right)\right\rangle=4 g^{2} R^{4} \omega^{4} D \delta\left(\chi-\chi^{\prime}\right)
$$

where we have assumed a delta function type of correlation with the strength $D$ for the random potential $\sigma(\chi)$. 当

The cumulative density of states $N(\omega)$ has the following form for the white noise potential with correlation given in ([II.30) [32].

$$
N(\omega)=\frac{\left(2 R^{2} \omega^{2} D\right)^{1 / 3}}{\pi^{2}} \frac{1}{A i^{2}\left(-(R \omega / 2 D)^{2 / 3}\right)+B i^{2}\left(-(R \omega / 2 D)^{2 / 3}\right)}
$$

where $A i$ and $B i$ are two independent Airy functions which satisfy the equation $y^{\prime \prime}-x y=0$.

The asymptotic behavior of the solution shows an exponential growth characterized by the Lyapunov exponent $\lambda_{\omega}$ defined by [6]

\footnotetext{
2 The "energy" $E=R^{2} \omega^{2}$ in (III.29) is dimensionless and so is the angular variable $\chi$.

${ }^{3}$ Hereafter we absorb $g$ in the definition of $D$
} 


$$
\lambda_{\omega}=\lim _{\chi \rightarrow \infty} \lambda_{\omega}(\chi)=\frac{1}{2 L_{l o c}(\omega)}
$$

where $L_{l o c}(\omega)$ is the localization length given below and the wave function grows as

$$
\psi(\chi) \rightarrow e^{\lambda_{\omega}(\chi) \chi}
$$

as $\chi$ increases.

In the case of white noise potential given in ([II.30), the Lyapunov exponent has the form 6.,

$$
\lambda_{\omega}=\frac{\sqrt{\pi}}{2} N(\omega) \int_{0}^{\infty} \sqrt{y} e^{-y^{2} / 12-(R \omega / 2 D)^{2 / 3} y} d y
$$

The entire curve is plotted in Fig.1. We obtain the asymptotic behavior of the localization length

$$
L_{l o c}(\omega) \sim \begin{cases}(D / R \omega)^{2 / 3} & \text { for } \omega \rightarrow 0 \\ 1 / D & \text { for } \omega \rightarrow \infty\end{cases}
$$

We see that in the long wavelength limit $\omega \rightarrow 0$, the wave function delocalizes. This is a direct consequence of the multiplicative nature of the stochasticity for classical wave propagation in random media [4].

\section{WAVE PROPAGATION IN SPACETIMES WITH TIME-DEPENDENT METRIC STOCHASTICITY: PARAMETRIC AMPLIFICATION AND PARTICLE CREATION}

Due to the conformal invariance of Maxwell's equations, time-dependent fluctuations in the scale factor can be transformed away (in a conformally-related coordinate). Assuming spatial homogeneity ,the time dependence of the metric is uniform in space, we start from the metric with the conformal fluctuations similar to ([II.17), but let the spatial part of the metric $f$ acquiring a time dependence. 
If the background spacetime curvature is negligible, the corresponding dielectric and peameability functions take the form

$$
\begin{aligned}
\epsilon_{i k} & =\mu_{i k}=h(\eta) \delta_{i k} \\
G_{i} & =0
\end{aligned}
$$

where we write the time-dependent factor as $h(\eta)$ instead of $f(\eta)$ for clarity. Assuming $h(\eta)$ has the exponential form as in (III.17)

$$
h(\eta)=e^{\nu(\eta)},
$$

then Maxwell's equations become

$$
\begin{aligned}
\nabla \times \vec{E} & =-\frac{d}{d \eta}(h(\eta) \vec{H}) \\
\nabla \times \vec{H} & =\frac{d}{d \eta}(h(\eta) \vec{E}) \\
\nabla \cdot \vec{E} & =0 \\
\nabla \cdot \vec{H} & =0 .
\end{aligned}
$$

From this we obtain a wave equation for $E$

$$
\nabla \times \nabla \times \vec{E}=-\frac{d}{d \eta}\left(h(\eta) \frac{d}{d \eta} h(\eta) \vec{E}\right),
$$

and a similar one for $\vec{H}$.

Since $\vec{E}$ has no divergence, for a slowly varying noise such that $|\dot{h}(\eta)|<<\omega h(\eta)$, where $\omega$ is the frequency of $\vec{E}$, the above equation takes the simpler form

$$
\frac{d^{2}}{d \eta^{2}} \vec{E}-\frac{1}{h^{2}} \nabla^{2} \vec{E}=0
$$

In momentum space, it reads

$$
\frac{d^{2}}{d \eta^{2}} \vec{E}_{k}+\frac{k^{2}}{h^{2}} \vec{E}_{k}=\frac{d^{2}}{d \eta^{2}} \vec{E}_{k}+k^{2} e^{-2 \nu(\eta)} \vec{E}_{k}=0 .
$$


Expanding in terms of $\nu$ gives

$$
\frac{d^{2}}{d \eta^{2}} \vec{E}_{k}+\left[k^{2}-2 \nu(\eta) k^{2}\right] \vec{E}_{k}=0
$$

This equation should be compared to ([11.29) with $j=0$ by identifying $V=2 \nu k^{2}$ and $E=k^{2}$.

Following the argument from ([II.29) to (III.35) and noting that the time coordinate $\eta$ plays the role of space coordinate $\chi$ here, we see the qualitative features of solutions to (IV.7): they show an exponential parametric amplification [33] with the rate characterized by the Lyapunov exponent $\lambda_{h}$ introduced in ([II.32)

$$
\lambda_{h}=\lim _{\eta \rightarrow \infty} \lambda_{h}(\eta)=\frac{1}{2 L_{h}(k)}
$$

and $\vec{E}_{k}$ grows

$$
\vec{E}_{k} \rightarrow \vec{E}_{k}(\eta=0) e^{\lambda_{h}(\eta) \eta}
$$

as $\eta$ increases. The characteristic time scale for parametric amplification $L_{h}(k)$ is equivalent to the localization length in ([II.32) under the $\eta \rightarrow \chi$ correspondence. For a Gaussian white noise $\nu$ with correlation $\left\langle\nu(\eta) \nu\left(\eta^{\prime}\right)\right\rangle=D \delta\left(\eta-\eta^{\prime}\right)$, the asymptotic behavior of $L_{h}(k)$ is given by

$$
L_{h}(k) \sim \begin{cases}(D / k)^{2 / 3} & \text { for } k \rightarrow 0 \\ 1 / D & \text { for } k \rightarrow \infty\end{cases}
$$

It is well-known that the cosmological particle creation problem in a FRW universe can be cast into an one-dimensional wave scattering problem [9] by reading the time variable $\eta$ as a space variable, and adopting the following boundary conditions:

$$
\vec{E} \rightarrow \begin{cases}e^{-i k \eta} & \text { for } \eta \rightarrow-\infty \\ \alpha e^{-i k \eta}+\beta e^{i k \eta} & \text { for } \eta \rightarrow \infty\end{cases}
$$


In this analogy, the reflection coefficient $\beta$ (or rather, $|\beta|^{2}$ )in the wave scattering picture gives the parametric amplification factor and, in second quantization, the particle creation rate. Since in this picture the transmission coefficient $t$ corresponds to the incoming flux which is normalized to one, the effect of localization translates to an exponential increase of the particle creation rate $\beta$ (so is $\alpha$ )

$$
\beta \rightarrow e^{\lambda_{h}(\eta) \eta} .
$$

More explicitly, if we compare (IV.11) with the corresponding scattering problem in which the wave packet is incident from $\eta=\infty$ and transit to $\eta=-\infty$

$$
\vec{E} \rightarrow \begin{cases}t e^{-i k \eta} & \text { for } \eta \rightarrow-\infty \\ e^{-i k \eta}+r e^{i k \eta} & \text { for } \eta \rightarrow \infty\end{cases}
$$

we see that the particle creation rate $N=|\beta|=|r / t|^{2}$ in expanding universe measures a dimensionless resistance in the wave transport picture. The transmission coefficient in a random potential is a multiplicative quantity which decreases exponentially with length. This implies that the logarithm of the resistance is an additive quantity and its average in length becomes constant in the thermodynamic limit and hence non-fluctuating. The average of the resistance itself (hence the average particle creation rate) is also known to show the exponential increase in length ( in conformal time $\eta$ ) with the rate characterized by $\lambda_{h}$. We can obtain the asymptotic behavior of the average of $N$ in the high energy limit as follows

$$
\langle N\rangle=\left\langle|\beta|^{2}\right\rangle=\frac{1}{2}\left(e^{2 \eta / L_{h}}+1\right) .
$$

Hence the fluctuations of $\langle N\rangle$ becomes

$$
\Delta N^{2}=\left\langle N^{2}\right\rangle-\langle N\rangle^{2}=\frac{1}{8} e^{4 \eta / L_{h}}\left(e^{2 \eta / L_{h}}-1\right)
$$


for $\eta>>L_{h}$ where $L_{h}=1 / D$ is the characteristic particle creation rate which appeared in (IV.8). The exponential divergence of $\Delta N^{2}$ results in the non-self-averaging nature of the observable.

\section{HAWKING RADIATION FROM A FLUCTUATING BLACK HOLE}

In this section, we study the effect of metric stochasticity in the Schwarzschild spacetime and discuss how it affects the Hawking radiation [10].

In the presence of metric stochasticity, the Helmholtz equation acquires a fluctuating component in the refractive index similar to the Friedmann-Robertson-Walker (FDR) universe case. Assuming the same conformal form of stochasticity as in ([II.17), but in a radial direction only for simplicity, the Helmholz equation in this case has the following form

$$
\frac{d^{2} \psi^{M}(r *)}{d r *^{2}}+\left[\omega^{2}+2 \sigma(r *) \omega^{2}-U(r *)\right] \psi^{M}(r *)=0
$$

where $\sigma(r *)$ is a stochastic variable as a function of $r *$. If we assume the metric stochasticity is restricted in the finite region $-L<r *<0$, the incoming flux from future infinity suffers backscattering not only from the potential $U(r *)$ but also from the randomness. The absorption probability $\Gamma_{\omega}$ is thus reduced by a factor of $e^{-\lambda_{\omega} L}$ where $\lambda_{\omega}$ is the Lyapunov exponent which appeared in (ㅍ․32) with $R=1$.

Hence the total luminosity has the form

$$
L=\frac{1}{2 \pi} \sum_{j=0}^{\infty}(2 j+1) \int_{0}^{\infty} d \omega \omega \Gamma_{0} e^{-\lambda_{\omega} L} /\left(e^{8 \pi M \omega}-1\right)
$$

The more interesting case is when the fluctuating region contains the event horizon such that the stochasticity induces horizon fluctuations [34 36]. For simplicity, we restrict the arguments to the scalar wave case in the rest of the section. The scalar wave in the metric (II.19) satisfies the equation 


$$
\left\{\left[\rho r(\rho)\left(1-\frac{2 M}{r(\rho)}\right)^{1 / 2} \frac{d}{d \rho}\right]^{2}+\omega^{2} r^{4}(\rho)\right\} \phi(\rho)=0 .
$$

This takes on a simple form if we define the coordinate $x$ such that $d x=f(\rho) d \rho / \rho^{2}$

$$
\left[\frac{d^{2}}{d x^{2}}+\omega^{2} r^{4}(x)\right] \phi(x)=0
$$

If the metric in (III.19) has the conformal type of stochasticity in the form $f_{1}(\rho) e^{2 \sigma(\rho)}$ and $f_{2}(\rho) e^{\nu(\rho)}$, where $\sigma(\rho)$ and $\nu(\rho)$ are two independent random variables with zero mean, the equation corresponding to $(\nabla .3)$ becomes

$$
\left\{\left[\rho r(\rho)\left(1-\frac{2 M}{r(\rho)}\right)^{1 / 2} e^{\sigma(\rho)-\nu(\rho)} \frac{d}{d \rho}\right]^{2}+\omega^{2} r^{4}(\rho)\right\} \phi(\rho)=0 .
$$

We can absorb $\sigma(\rho)$ by choosing the coordinate $x$ such that $d x=f(\rho) e^{\nu(\rho)-\sigma(\rho)} d \rho / \rho^{2}$. Then we have

$$
\left[\frac{d^{2}}{d x^{2}}+\omega^{2} r^{4}(x) e^{4 \nu(\rho)}\right] \phi(x)=0
$$

At the horizon, only incoming wave exists. So the solution of (․6) will be

$$
\phi \sim \exp \left[-i \omega r_{s}^{2} e^{4 \nu\left(\rho_{s}\right)} x\right]
$$

where $r_{s} \equiv 2 M$ and $\rho_{s} \equiv M / 2$. This mode function behaves as

$$
\begin{aligned}
\phi & \rightarrow 1-i \omega r_{s}^{2} e^{4 \nu\left(\rho_{s}\right)} x \\
& \rightarrow 1+\frac{i \omega r_{s}^{2} e^{4 \nu\left(\rho_{s}\right)}}{\rho}
\end{aligned}
$$

as $x \rightarrow 1$ or, equivalently, $\rho \rightarrow \infty$. Here we assumed the stochastic variables $\sigma(\rho)$ and $\nu(\rho)$ vanish at infinity.

Far from the horizon, the asymptotic flatness of the spacetime allows us to write (V.3) as

$$
\left\{\left[\rho r(\rho) \frac{d}{d \rho}\right]^{2}+\omega^{2} r^{4}(\rho)\right\} \phi(\rho)=0
$$


with the solution

$$
\begin{aligned}
\phi(\rho) & =\frac{1}{\sqrt{\omega \rho}}\left[A J_{1 / 2}(\omega \rho)+B J_{-1 / 2}(\omega \rho)\right] \\
& \rightarrow \sqrt{\frac{2}{\pi}}\left[A+\frac{B}{\omega \rho}\right] \quad \text { for } \omega \rho<<1 .
\end{aligned}
$$

Comparing (V.10) with (V.8), we obtain the following absorption coefficient for low energy scattering of the scalar wave,

$$
\begin{aligned}
\Gamma_{a} & =1-\left|\frac{1+i \frac{B}{A}}{1-i \frac{B}{A}}\right|^{2} \\
& \rightarrow 4 \omega^{2} r_{s}^{2} e^{4 \nu\left(\rho_{s}\right)} \quad \text { for } \omega \rightarrow 0 .
\end{aligned}
$$

From $(\nabla .11)$, the absorption cross section for this process is

$$
\begin{aligned}
\sigma_{a} & =\frac{\pi}{\omega^{2}} \Gamma_{a} \\
& \rightarrow 4 \pi r_{s}^{2} e^{4 \nu\left(\rho_{s}\right)} \quad \text { for } \omega \rightarrow 0 \\
& =4 \pi r_{s}^{2}\left[1+4 \nu\left(\rho_{s}\right)+8 \nu^{2}\left(\rho_{s}\right)+\cdots\right]
\end{aligned}
$$

The average absorption cross section is then

$$
\begin{aligned}
\left\langle\sigma_{a}\right\rangle & =4 \pi r_{s}^{2}+32 \pi r_{s}^{2}\left\langle\nu^{2}\left(\rho_{s}\right)\right\rangle \\
& =A_{H}+8 A_{H}\left\langle\nu^{2}\left(\rho_{s}\right)\right\rangle
\end{aligned}
$$

where $A_{H} \equiv 4 \pi r_{s}^{2}$ is the area of the event horizon and assume all the higher moments of $\nu$ vanish. It is known that low energy absorption cross section of the scalar wave gives the area of the event horizon for a large class of black hole solutions 38].

Therefore, we expect the second term gives the horizon area fluctuations

$$
\delta A_{H}=8 A_{H}\left\langle\nu^{2}\left(\rho_{s}\right)\right\rangle
$$

which induces fluctuations in the Hawking temperature

$$
\frac{\delta T_{H}}{T_{H}}=4\left\langle\nu^{2}\left(\rho_{s}\right)\right\rangle
$$




\section{DISCUSSION}

In this paper, we have studied the effect of metric fluctuations on wave propagation in flat and curved spacetimes. We saw that for electromagnetic waves the effect of metric stochasticity is equivalent to that of a random optical index. With this analogy, we can calculate the correction to the scattering cross section of the electromagnetic wave in curved spacetime with metric fluctuations. We also see the intensity fluctuations due to multiple scattering. If we assume classical spacetime is effectively emerging from the underlying quantum fluctuations by coarse graining, it seems reasonable to assume that the stochastic part reflects the symmetry of the background spacetime. When the stochastic part of the metric has only radial dependence, the problem is further reduced to an one dimensional transport problem in which Anderson localization is manisfest. For time-dependent but space-independent stochasticity, particle creation rate shows exponential instability in conformal time. Furthermore, analogy with mesoscopic transport in one dimension predicts large fluctuations in the particle creation rate due to its non-self-averaging nature. For Schwarzschild spacetime, time-independent stochasticity induces fluctuations of the event horizon and ,correspondingly, Hawking temperature.

The restrictions we made on separate space and time dependence of the stochastic metric is mainly for technical simplicity. We expect qualitatively similar phenomenon in the more general cases, where space- and time -dependent metric fluctuations both exist. Certain modifications are expected such as dissipative effects on localization for a time-dependent random potential.

Possible origins of the metric fluctuations have been discussed recently. Primordial

stochastic gravitational waves is currently under intense investigation [23, 39, 40]. Stochasticity used in such a context is different from that in stochastic semiclasical gravity theory 12,16 18. In the former it refers to (quantized) linear perturbation of the metric obeying 
Einstein's equations whereas in the latter, metric fluctuations is induced by fluctuations in quantum matter fields, which obey the Einstein-Langevin equation, as a generalization of the semiclassical Einstein equation governing the mean values of metric and matter stress energy. The naive replacement of the stress-energy tensor with its expectation value leads to some pathological results such as the violation of the weak energy condition of the Einstein equation [13]. Possible cure may be obtained by including the smearing term in the classical background spacetime [15]. Self-consistency between the classical gravitation and the quantum matter sectors also points to the inevitable dynamic role of metric and field fluctuations [11]. From the astrophysical viewpoint, cosmic string network may provide the source of the stochastic gravitational wave background which lies in the observable frequency range of the LIGO detector [23]. The discussions and results in this work are more of a generic nature insensitive to the origin of particular sources.

Ford argued that the lightcone fluctuations based on linearized quantum gravity induce black hole horizon fluctuations [34]. He suggests that the horizon fluctuations are small enough to validify the semiclassical derivation of Hawking radiation. Casher, et. al. argue that the semiclasscal approximation breaks down close to but much larger than the Planck scale from the horizon [36] due to the interaction with the atmosphere of the horizon. Sorkin's argument is based on Newtonian mechanics and its vadility for general relativistic cases remains to be shown [35]. Since we assumed a static metric stochasticity for the Schwarzschild spacetime, direct comparizon with their results is not obvious. Nevertheless, we believe our arguments have certain degrees of generality, knowing that the randomness can arise from the imhomogeneity of a static spacetime. These issues are currently under investigation.

Acknowledgement We thank Prof. P. Sheng for introducing us to the subject of wave propagation in a random media, and Prof. E. Calzetta for useful comments. We enjoyed the hospitality of the physics and mathematics department of the Hong Kong University of 
Science and Technology where part of this work was done. This work is supported in part by the U S National Science Foundation under grants PHY94-21849.

[1] P. W. Anderson Phys. Rev. 109, 1492 (1958).

[2] H. L. Frish, in Probabilistic Methods in Applied Mathematics (Academic Press, New York, 1968).

[3] A. Ishimaru, Wave Propagation and Scattering in Random Media (IEEE press, New York, 1997).

[4] S. John, in Photonic Band Gaps and Localization eds C. M. Soukoulis (Plenum Press, New York, 1993). P. Sheng, Introduction to Wave Scattering, Localization, and Mesoscopic Phenomena (Academic Press, New York, 1995).

[5] P. A. Lee and T. V. Ramakrishnan, Rev. Mod. Phys. 57, 287 (1985).

[6] I. M. Lifshits, S. A. Gredeskul, and L. A. Pastur, Introduction to the theory of Disordered Systems (John Wiley \& Sons, Inc. 1988).

[7] C. W. Misner, K. S. Thorne, and J. A. Wheeler, Gravitation (W. H. Freeman and Company, New York, 1973).

[8] N. D. Birrell, P. C. Davis, Quantum Fields in Curved Space (Cambridge University Press, New York, 1982).

[9] L. Parker, Phys. Rev. 183, 1057 (1969); R. U. Sexl and H. K. Ubantke, Phys. Rev. 179, 1247 (1969); Ya. B. Zeldovich, Zh. Eksp. Teor. Fiz. Pis'ma Red. 12, 443 (1970) [JETP Lett.32, 307 (1970)]; B. L. Hu, Phys. Rev. D 9, 3263 (1974); B. S. De Witt, Phys. Rep. C19, 295 (1975). 
[10] S. W. Hawking, Nature 248, 30 (1974); Com. Math. Phys. 43, 199 (1975).

[11] B. L. Hu, Physica A158, 399 (1979).

[12] E. Calzetta and B. L. Hu, Phys. Rev. D49, 6636 (1994).

[13] C. I. Kuo and L. H. Ford, Phys. Rev. D47, 4510 (1993).

[14] N. Phillips and B. L. Hu, Phys. Rev. D 55, 6123 (1997).

[15] E. Flanagan and R. M. Wald, Phys. Rev. D54, 6233 (1996).

[16] B. L. Hu and A. Matacz, Phys. Rev. D51, 1577 (1995).

[17] B. L. Hu and S. Sinha, Phys. Rev. D51, 1587 (1995).

[18] A. Campos and E. Verdaguer, Phys. Rev. D53, 1927 (1996).

[19] E. Calzetta, A. Campos, and E. Verdaguer, Stochastic Semiclassical Cosmological Models, grqc/9704010.

[20] B. L. Hu, N. Phillips and A. Raval, Fluctuations of the Energy Mementum Tensor of a Quantum Field in a Black Hole Spacetime (in preparation)

[21] A. Campos, B. L. Hu and A. Raval, Fluctuation-Dissipation Relation for a Quantum Black Hole in Quasi-equilibrium with its Hawking Radiation (in preparation)

[22] B. L. Hu, Alpan Raval and S. Sinha, Fluctuation-Dissipation Relation for a Quantum Black Hole with Self-consistent Quasi-static Backreaction (in preparation)

[23] B. Allen,Stochastic Gravitational Wave Background, gr-qc/9604033, to be published in the proceedings of Astrophysical Sources of Gravitational Radiation, Les Houches, France, 26 Sep - 6 Oct 1995, and reference therein.

[24] B. Mashhoon Phys. Rev. D8, 4297 (1973); J. Plebanski Phys. Rev. 118, 1396 (1960); A. M. 
Volkov, A. A. Izmest'ev, and G. V. Skrotskii, Zh. Eksp. Teor. Fiz. 59, 1254 (1970) Sov. Phys. - JETP 32, 686 (1971).

[25] B. Mashhoon, Phys. Rev. D7, 2807 (1973).

[26] E. M. Lifshitz, J.Phys., U.S.S.R., 10, 116 (1946).

[27] R. Balian and J-J Niez, J. Phys. I France, 5, 7 (1995).

[28] L. Parker, Phys. Rev. 183, 1057 (1969).

[29] B. Shapiro, Phys. Rev. Let. 57, 2168 (1986).

[30] M. J. Stephen and G. Cwilic, Phys. Rev. Lett. 59, 285 (1987).

[31] R. A. Webb, S. Washburn, C. P. Umbach, and R. B. Laibowitz, Phys. Rev. Let. 54, 2696 (1985); P. A. Lee, A. D. Stone and H. Fukuyama, Phys. Rev.B35, 1039 (1987).

[32] B. I. Halperin, Phys. Rev. 139, 104 (1965).

[33] Ya. B. Zeldovich, Zh. Eksp. Teor. Fiz. Pis'ma Red. 12, 443 (1970) - JETP Lett.32, 307 (1970).

[34] L. H. Ford, Cosmological and Black Hole Horizon Fluctuations, gr-qc/9704050.

[35] R.D. Sorkin, How Wrinkled is the Surface of a Black Hole?, gr-qc/9701056.

[36] A. Casher, F. Englert, N. Itzhaki, and R. Parentani, Black Hole Horizon Fluctuations, hepth/9606106.

[37] L. Parker, Phys. Rev. D12, 1519 (1975).

[38] W. G. Unruh, Phys. Rev. D14, 3251 (1976); A. A. Storobinsky and S. M. Churilov, Sov. Phys. JETP 38, 1 (1974); S. R. Das, G. Gibbons, S. D. Mathur, Phys. Rev. Let. 78, 417 (1997).

[39] L. H. Ford, Phys. Rev. D51, 1692 (1995). 
[40] L. P. Grishchuck and Y. V. Sidorov, Phys. Rev. D42, 3413 (1990).

Figure 1 The localization length is plotted as a function of frequency. R and D are set to 1 . 